\section{Cahiers de Narratologie}

Analyse et théorie narratives

$10.1 \mid 2001$

La voix narrative

\title{
Savoir retenu et savoir manquant. Quelques enjeux de la narration omnisciente dans le récit contemporain
}

Frances Fortier et Andrée Mercier

\section{CpenEdition}

Journals

Electronic version

URL: http://journals.openedition.org/narratologie/6976

DOI: 10.4000/narratologie.6976

ISSN: 1765-307X

Publisher

LIRCES

\section{Printed version}

Date of publication: 1 January 2001

Number of pages: $445-460$

ISBN: 2914561032

ISSN: 0993-8516

Electronic reference

Frances Fortier et Andrée Mercier, "Savoir retenu et savoir manquant. Quelques enjeux de la narration omnisciente dans le récit contemporain", Cahiers de Narratologie [Online], 10.1 | 2001, Online since 19 November 2014, connection on 23 February 2021. URL: http://journals.openedition.org/narratologie/ 6976 ; DOI: https://doi.org/10.4000/narratologie.6976 


\title{
SAVOIR RETENU ET SAVOIR MANQUANT. QUELQUES ENJEUX DE LA NARRATION OMNISCIENTE DANS LE RÉCIT CONTEMPORAIN.
}

\author{
Frances FORTIER et Andrée MERCIER \\ Université du Québec \\ Université Laval, Canada
}

$\mathrm{Au}$ nombre des caractéristiques de la production narrative contemporaine (plus exactement celle des deux dernières décennies), le recours marqué aux formes de l'intime et, plus largement, la prise en charge du récit par une parole subjective, celle d'un ou de multiples "je », sont fréquemment signalés par la critique'. Par ses hésitations mêmes, par ses glissements et manifestations polyphoniques, la voix narrative se fait ainsi envahissante. Plus discrète, la narration omnisciente (entendons ici, provisoirement, un narrateur hétérodiégétique, non représenté, à focalisation zéro, pour reprendre la terminologie de Genette) occupe toujours une partie du territoire narratif récent, soucieux d'invention formelle. C'est cette catégorie de textes, à la voix narrative ténue, que veut aborder notre étude.

1 Mentionnons, pour la littérature française, les travaux de Dominique Viart, notamment "Mémoires du récit : questions à la modernité » dans Dominique Viart (dir.), Mémoires du récit, ParisCaen, Lettres modernes Minard, 1998, p. 3-27 (Coll. «La revue des lettres modernes ", série "Écritures contemporaines "). Sur la littérature québécoise, des observations similaires ont été faites. Signalons, parmi bien d'autres études, l'article de Lise Gauvin, "Introduction. L'âge de la prose : romans et récits des années 80 », dans Lise Gauvin et Franca Marcato-Falzoni dir., L'âge de la prose. Romans et récits québécois des années 80 , Montréal/Rome, VLB éditeur/Bulzoni editore, 1992, p. 9-17. 
Comme le rappelle Cécile Cavillac, dans un article intitulé «Vraisemblance pragmatique et autorité fictionnelle » ${ }^{2}$, la narration omnisciente est une convention qui, pour aller de soi pour les lecteurs de fiction que nous sommes, était pourtant irrecevable jusqu'aux premières décennies du XIX ${ }^{\mathrm{e}}$ siècle. Ce droit de connaître des faits que nul témoin ne peut attester, d'accéder aux pensées les plus intimes des personnages, n'était pas admis. Ne pouvant se permettre d'agir comme un fabulateur, le narrateur se voyait soumis à un strict protocole pragmatique et devait recourir à toutes sortes d'expédients pour justifier sa connaissance des faits ${ }^{3}$. Cette obligation conduira par exemple les romans de chevalerie à jouer d'un " paradoxe naïvement retors $»^{4}$ comme le souligne Cavillac : plus les faits racontés sont extraordinaires, merveilleux, plus ils risquent d'être connus. Autrement dit plus l'histoire est empiriquement invraisemblable, plus la vraisemblance pragmatique sera sauve : le narrateur est alors tout à fait justifié d'avoir eu écho de tels événements. C'est dire qu'à l'inverse, les faits et les êtres ordinaires, "la sphère du privé, du personnel, de l'humble, de l'obscur $»^{5}$, se heurtent à la difficulté de leur mise en récit.

L'omniscience est désormais recevable et le narrateur, du moment qu'il est hétérodiégétique et non représenté dans le récit, se voit reconnaître d'emblée par le lecteur une autorité absolue en matière de savoir ${ }^{6}$. Cette convention semble

2 Cécile CAVILLAC, «Vraisemblance pragmatique et autorité fictionnelle ", Poétique, n 101, février 1995, p. 23-46.

${ }^{3} \mathrm{C}$ 'est bien sûr une certaine conception de la fiction qui détermine les conditions de recevabilité des modes de narration. « Dès l'époque médiévale, le récit de fiction est conçu comme une imitation de la seule réalité que puisse imiter le langage, à savoir le langage lui-même. Il est censé reproduire non la vie, mais les «dits" sur la vie». Cécile Cavillac, ibid., p. 24.

${ }^{4}$ Ibid.

5 Ibid., p. 27.

6 Pour reprendre les propos de Genette : «En fiction, le narrateur hétérodiégétique n'est pas comptable de son information, 
pourtant mise à mal dans certains textes contemporains. En effet, sous le couvert de l'omniscience, des narrateurs gèrent de façon singulière leur compétence cognitive et conduisent le lecteur à l'hypothèse d'un savoir retenu, omis ou, même, franchement ignoré. Le pacte tacite, selon lequel le narrateur omniscient possède une connaissance illimitée de la réalité narrée et doit livrer l'information nécessaire à la compréhension de l'histoire, ne semble plus respecté. Nous nous en tiendrons ici à deux manifestations différentes de ce mode de narration. Le premier exemple, le roman Un an de l'écrivain Jean Echenoz ${ }^{7}$, semble associer la narration omnisciente à un jeu délibéré de mystification du lecteur. Le deuxième exemple, le bref récit Anna à la lettre $C$, de l'écrivain québécois Larry Tremblay ${ }^{8}$, se présenterait plutôt comme un exercice de démystification. Nous verrons que, chacun à leur façon, ils témoignent de ce que Dominique Viart a pu qualifier d'une « reviviscence des formes narratives ${ }^{9}$, d'une remise à l'honneur du récit et du sujet mais traversés par un «doute radical». Bref nous verrons comment ils relancent l'omniscience et jouent d'une convention gagnée par le soupçon.

Le savoir retenu ou la mystification délibérée chez Echenoz

On connaît bien la manière Echenoz : détournement des modèles romanesques, profusion de descriptions, personnages à l'étrangeté délirante, métalepses et clins d'œil textuels créent un univers singulier, où la maîtrise de l'écriture sert une ironie malicieuse ${ }^{10}$. Avec Un an, son avant-dernier roman,

l'« omniscience » fait partie de son contrat [...] ». Nouveau discours $d u$ récit, Paris, Seuil, 1983, p. 52.

7 Jean ECHENOZ, Un an, Paris, Éd. de Minuit, 1997, 110 p.

8 Larry TREMBlay, Anna à la lettre $C$, Montréal, Éd. Les Herbes rouges, $1992,52 \mathrm{p}$.

9 Dominique VIART, op. cit., p. 7.

10 Signalons le livre de Jean-Claude Lebrun, entièrement consacré à l'œuvre d'Echenoz: Jean Echenoz, Monaco, Ed. du Rocher, 1992 (coll. «Domaine français »). Ajoutons le plus récent ouvrage consacré en partie à l'œuvre d'Echenoz: Bruno Blanckeman, Les récits indécidables: Jean Echenoz, Hervé Guibert, Pascal Quignard, 
Echenoz nous replonge dans ce cadre familier, mais cette fois avec un tour de plus. Dans ce « road story » mâtiné de polar, une jeune femme en fuite, Victoire, erre pendant près d'un an dans le sud-ouest de la France, de peur qu'on ne l'accuse du meurtre de son compagnon Félix qu'elle a trouvé mort auprès d'elle un matin de février. Son périple, ponctué des visites impromptues de Louis-Philippe, un ami commun qui l'informe des développements de l'affaire, s'achève sur un coup de théâtre : rentrée à Paris à l'instigation de Louis-Philippe - qui lui affirme que tout danger est écarté - Victoire se retrouve nez à nez avec un Félix bien vivant, qui lui apprend, ô surprise, le suicide, déjà vieux de près d'un an, dudit LouisPhilippe. Le lecteur, médusé par cette entourloupette - le mort est en pleine forme alors que le vivant est tout à fait mort n'a d'autres ressources que de reprendre sa lecture depuis le début pour tenter de déceler la présence d'indices qui auraient pu laisser entrevoir un tel dénouement. Cette relecture le laissera sur sa faim : il a bel et bien été berné par le narrateur de l'histoire, qui n'a jamais laissé entrevoir la possibilité de cette finale en queue de poisson défiant toute logique narrative ${ }^{11}$.

Voyons voir le statut de ce narrateur, qui avait pourtant instauré un régime de confiance, tant par la minutie de sa narration que par ses intrusions inopinées. Non représenté dans l'histoire qu'il raconte en narration ultérieure, ce narrateur suit à la trace tous les déplacements de Victoire : d'abord de la gare Montparnasse à la gare de Bordeaux, où elle hésite entre trois destinations (Saint-Jean-de-Luz, Auch et Bagnères-de-Bigorre) pour choisir la première ; viendront ensuite, ses finances péréclitant, Mimizan-Plage, toute la région des Landes qu'elle parcourt à vélo, le triangle Arcachon, Nérac et Dax, en passant par Onesse-et-Laharie et Trensacq, où elle se fait voler sa bicyclette; poursuivant son

Villeneuve d'Ascq, Presses universitaires du Septentrion, 2000 (Coll. «Perspectives »).

11 Comme le signale Dominique Viart, «Echenoz, [...], ne refuse rien à la narration, laquelle se poursuit parfois au péril même de la cohérence diégétique ». Op. cit., p. 18. 
périple en auto-stop, Victoire, toujours sous le regard du narrateur, rejoint la gare de Toulouse-Matabiau d'où, en compagnie de deux comparses (Gore-Tex et Lampoule), elle repart vers les Landes ; un larcin qui se termine mal la laissera mal en point, dans un fossé, où elle sera recueillie par deux marginaux (Castel et Poussin); elle passera l'été en leur compagnie, puis s'enfuira à nouveau pour rejoindre Bordeaux, et, de là, toujours en stop, regagner Paris. Son périple s'achève à pied, jusqu'à la gare Saint-Lazarre cette fois. La boucle est bouclée, Victoire est rentrée. L'attention prêtée au référent spatial, conjuguée à la description minutieuse de la déchéance progressive de Victoire - qui passe du statut de jeune Parisienne branchée à celui d'itinérante solitaire sale et dépenaillée, jouant les simples d'esprit confirment l'omniscience et le rôle d'observateur du narrateur.

Quelques intrusions métaleptiques, nous sommes bien chez Echenoz, viennent en outre appuyer son statut de régisseur, même si ce statut est parfois "ironisé », comme dans cet extrait où le narrateur, à la faveur du ON, se montre littéralement à la remorque de son personnage :

Cette route est loin des plages, on ne voit pas la mer, on le regrette. On aimerait bien regarder naître et grossir les vagues et se renverser, voir indéfiniment chacune d'elle décliner sa version, son interprétation de la vague idéale [on aura reconnu le Palomar de Calvino], on pourrait comparer leur allure, leur conception, leur succession, leur son, mais non, Victoire descendit du car vers quinze heures à Mimizan. (p. 48).

Équivoque, cet accroc à l'habituelle discrétion et toutepuissance du narrateur omniscient sert aussi à rappeler les règles du genre et à y souscrire car, pour assurer la bonne marche du récit, le narrateur entend bien, en effet, suivre les pérégrinations de son personnage et non ses propres envies... L'instance narrative assure encore sa «fiabilité » de diverses manières. En ponctuant le texte d'anticipations qui la montrent bien au fait de la suite de l'histoire («Victoire proposa de régler en liquide, sur-le-champ, si le logement lui convenait. Il lui conviendrait. Elle y passerait trois mois » 
p. 14) ou en neutralisant dès le début les premières aspérités logiques ("Quiconque, à la place de Victoire, eût en pareil cas pris conseil de ses proches mais pas elle, sans famille et tout pont coupé ", p. 10), cette instance narrative à la présence discrète mais affirmée, apparaît bien comme celle qui tire les ficelles. Il n'est jusqu'au NOUS rhétorique qui n'a de cesse d'affirmer, de manière certes paradoxale, la présence d'un narrateur omniscient. Ce NOUS de connivence recouvre non plus, comme Echenoz nous y avait habitué, le lien entre le narrateur et le lecteur mais désigne cette fois le rapport entre le narrateur et sa protagoniste :

$\mathrm{Au}$ pire elle finirait toujours par décrocher quelque emploi de vendeuse ou de caissière, trouver quelque amant moins indélicat que Gérard [...] nous verrions. Nous n'étions pas pressée. Nous n'envisagerions ce point vraiment qu'en toute dernière extrémité. En attendant nous prîmes une chambre à l'hôtel Albizzia. (p. 46)

Cet hapax dans la narration pourtant hétérodiégétique est d'autant plus pervers qu'il s'accompagne de délégations de la focalisation qui viennent confirmer, en quelque sorte, le fait que le texte est non focalisé (Genette parlerait de focalisation zéro). Nous ne retiendrons qu'un seul exemple, celui où Victoire nous est montrée en position de focalisatrice, " accoudée à la barre d'appui de sa fenêtre », en train de considérer la mer vide (p. 28) ; un cargo étant apparu dans le lointain, l'intérêt de Victoire semble alors porter sur le radiotélégraphiste, "accoudé au bastingage », qui " considérait dans sa longue-vue la côte pointillée de pavillons » (p. 28), jusqu'à ce que la sonnerie de la porte suspende la contemplation de la jeune femme. Du narrateur à Victoire puis au radiotélégraphiste, avec retour progressif vers le narrateur, la focalisation bouge, indexant ici, de manière ironique, des jeux de regard qui accusent la gestion singulière du savoir de ce narrateur toujours omniscient. En effet, Victoire, à trop grande distance de la mer $^{12}$, ne saurait apercevoir le radio-télégra-

12 Comme il est précisé quelques pages auparavant : «quoique visible, l'océan était trop éloigné pour qu'on pût l'entendre » (p. 23). 
phiste. Quant à la description minutieuse de ce qu'observe le radio-télégraphiste grâce à sa longue-vue, elle détourne habilement des pensées de Victoire. Cette pseudo-délégation de la focalisation aux personnages n'en cache donc pas moins l'emprise du narrateur, qui, si l'on veut reprendre une formulation de Genette, «place [...] son «foyer » en un point si indéterminé, [...], qu'il ne peut coïncider avec aucun personnage $[\ldots] »^{13}$. Ainsi, chez Echenoz, le regard oscille et, s'il se fixe, ce sera souvent sur des détails insignifiants, regard maniaque d'une omniscience en quelque sorte détournée de son rôle habituel de sélection et d'interprétation.

D'autres extraits témoignent encore d'un code de focalisation délibérément perturbé. Là où on aurait pu croire à une vision « binoculaire » également partagée entre Victoire et le narrateur, on retrouve plutôt une mise en scène de l'incompétence «focalisatrice » de Victoire. Ainsi en est-il des innombrables intérieurs de voiture, minutieusement décrits (on se souviendra que Victoire se déplace en auto-stop); une grosse Renault, un fourgon mortuaire, une R5 sans option, qui sent le chien sans chien, une Séat, un vieux modèle de Ford Escort, qui sent le chien avec chien, la prendront à bord tour à tour :

[...] outre leur personnalité, Victoire observait la marque, la couleur et l'aménagement de leur véhicule qui l'avançait vers un but mal déterminé. Les premiers temps elle était attentive à ces détails, elle finit par y prendre de moins en moins garde. (p. 64)

Alors que Victoire délaisse la perception, le narrateur prend le relais en imitant en quelque sorte le regard particulier de celle-ci ${ }^{14}$ : viendront ainsi une vieille 505 avec chien mais

$13 \mathrm{Op}$. cit., p. 49. Si l'on considère toute la surface d'un récit, la focalisation zéro combinerait toutefois plus exactement "une mosaïque de segments diversement focalisés " (p. 49), mais dont certains ne pourraient être rattachés au regard d'un personnage ou d'un observateur situé en un point déterminé de la diégèse.

14 Bruno BLANCKEMAN s'est intéressé à la ventriloquie du narrateur echenozien, à sa façon d'imiter différentes voix sans déléguer explicitement la parole. Voir ici même son article intitulé 
sans odeur de chien, une Saab ardoise, une petite Fiat blanche (celle de Louis-Philippe), une Simca Horizon (celle de Gérard). Fragmenté, hachuré, le parcours de Victoire est une fuite sans but, ou, pour reprendre les mots mêmes du texte, « une errance en dents de scie, pas très contrôlée [...] s'apparentant plutôt au trajet brisé d'une mouche enclose dans une chambre » (p. 63). Victoire, à la différence du narrateur, dirions-nous malicieusement, ne maîtrise pas son univers ; son incompétence sert toutefois très précisément la cause du narrateur.

À preuve, l'épisode des balles de golf qui retiendra ici notre attention par sa double incidence sur la narration et sur l'écriture. Sur le plan narratif, les trois séquences consacrées aux balles de golf traduisent l'incompétence cognitive de Victoire - en même temps que la nôtre - tout en permettant la progression de l'histoire. Une première séquence pose l'énigme (p. 23) : " des sons légers, à peine audibles, de nature et d'amplitude variables, étouffés ou mats ", attirent l'attention de Victoire « qui finit par se mettre à l'affût sans pouvoir établir leur origine »; une seconde séquence réactualise les " chocs discrets déjà repérés [...] Mais pas plus que les fois précédentes elle ne parvint à déterminer-leur origine » (p. 31) ; il faudra qu'une balle de golf perfore la glace arrière du véhicule de Louis-Philippe pour que Victoire comprenne enfin « l'origine des bruits anonymes qui l'intriguaient depuis son arrivée » (p. 37). Aurons-nous besoin d'un tel fracas pour comprendre l'enjeu du texte alors que le narrateur nous avait pourtant précisé que les fenêtres arrière du pavillon où Victoire s'était réfugiée donnaient sur un terrain de golf... (p. 14) ? Cette amorce de métaphore du lecteur intrigué - qui entend les bruits discrets du texte mais qui aura besoin d'une fin fracassante pour en saisir l'enjeu - reviendra clore le récit : la description d'un boulevard bordé d'arbres d'où « tombent des marrons qui rebondissent parfois sur les voitures en négatifs de balles de golf » (p. 109) signale le statut d'un texte construit avant tout comme un exercice d'écriture. Avec insistance, le texte rapproche le «trou circulaire » formé

«Jean Echenoz ou le récit ventriloque». La ventriloquie trouverait donc son équivalent sur le plan de la focalisation. 
par la balle de golf du «trou circulaire » pratiqué dans la vitre du guichet de la gare Saint-Lazarre :

Il est surprenant, dira le texte, que ce trou circulaire dans la vitre, conçu pour transmettre des informations strictement ferroviaires et d'un contour plus régulier que celui que Victoire a vu se former, six mois plutôt, sur la voiture de Louis-Philippe, puisse véhiculer de tels points de vue sans que tout le système explose (p. 109)

Ce commentaire métatextuel attire l'attention sur le « trou » de la narration : à la page suivante, Victoire apprendra, et nous avec elle, que Félix est vivant et que le LouisPhilippe qu'elle a rencontré à plusieurs reprises était bel et bien mort. Il faudra attendre le roman suivant d'Echenoz, $J e$ m'en vais (qui s'est mérité le Goncourt 1999) pour connaître le fin mot de l'histoire. Félix, devenu Félix Ferrer, avait été victime d'un accident cardiaque (du bloc auriculo-ventriculaire) : c'est parce qu'elle avait pris cet endormissement des fonctions vitales pour la mort que la Victoire d'Un an s'était enfuie. De même, les apparitions inexplicables de LouisPhilippe s'éclairent à la lecture de Je m'en vais: LouisPhilippe Delahaye, l'assistant de Félix - qui lui avait même présenté Victoire - avait maquillé sa propre disparition en suicide (et changé son nom en Baumgartner) pour masquer une malversation et fuir en Espagne, en empruntant le même trajet que la Victoire d'Un an.

Par le biais des liens intratextuels amorcés ou par les clins d'œil qui renvoient à des textes antérieurs - les descriptions de voitures, l'allusion à la mouche, les métalepses narrativesle narrateur incarne de mieux en mieux la figure de l'auteur Echenoz. Si Un an joue bien la carte de la narration hétérodiégétique à focalisation zéro, s'il utilise bien la conception conventionnelle de la narration omnisciente pour duper son lecteur, il faut bien voir cependant que cette stratégie, ludique à souhait mais non gratuite, est au service du style de l'œuvre, d'une écriture. Toujours aux commandes, par l'entremise d'un narrateur qui multiplie les angles de vision, change de focale pour mieux retenir son savoir, Jean Echenoz, comme il le dit lui-même 
est à la fois le narrateur et l'auteur, c'est-à-dire moi, peut-être un peu plus que pour d'autres, je, [puisque] J. E. ce sont mes initiales. (Paroles rapportées par JeanBaptiste Harang, "Jean Echenoz, Arctique de Paris », Libération, 16 septembre 1999)

Nul doute, en effet, que le détournement de la narration omnisciente s'exerce ici au profit de l'expression d'une autorité et d'une subjectivité de plus en plus fortes qui sollicitent la participation du lecteur.

Le savoir manquant ou l'omniscience démystifiée chez Tremblay

L'exemple du récit de Larry Tremblay ${ }^{15}$ nous intéresse en ce qu'il montre un usage particulier de la narration omnisciente mis, cette fois, au service d'un genre. Anna à la lettre $C$ appartient en effet à un ensemble générique qui, au Québec notamment, a pris un essor très net depuis 1980: le récit ${ }^{16}$. Le récit partage avec le roman contemporain une même fascination pour la subjectivité et les territoires de l'intime. Il s'y engage toutefois de façon plus nette. Évitant la mise en intrigue au profit d'une succession événementielle qui ne révèle pas clairement une logique de cause à effet, le récit s'accompagne souvent d'un mode d'énonciation éminemment subjectif : la narration omnisciente y est, de fait, assez rare. Le registre retenu sera plutôt celui de la voix unique, le «je », ou encore celui de la cohabitation de voix sans véritable interaction, comme si le « je » ne pouvait établir

15 Également poète, essayiste et dramaturge, Larry Tremblay, a signé, entre autres pièces, The Dragonfly of Chicoutimi (Montréal, Éd. Les herbes rouges, 1995) et Le génie de la rue Drolet (CarnièresMorlanwelz, Éd. Lansman, 1997).

16 Voir à ce sujet le numéro de revue québécoise préparé par Frances Fortier et Andrée Mercier, "Le récit littéraire des années quatre-vingt et quatre-vingt-dix », Voix et Images, $n^{\circ} 69$, printemps 1998, où on lira entre autres tant l'esquisse du portrait sociologique du genre (Frances Fortier et René Audet, "Le récit, émergence d'une pratique : le volet institutionnel », p. 439-460) qu'une mise au jour de ses traits constitutifs (Andrée Mercier, "Poétique du récit contemporain : négation du genre ou émergence d'un sous-genre ?», p. 461480). 
que son propre témoignage, sans pouvoir jamais le dépasser et rejoindre le dialogue. Il en découle une vision partiale, mais surtout partielle, des événements racontés ${ }^{17}$.

Si le recours à la narration omnisciente tend à démarquer Anna à la lettre $C$ des formes convenues du récit, le texte de Larry Tremblay a en commun avec le reste du genre une histoire banale, un nombre réduit de personnages, une structure temporelle simple et un regard subjectif ${ }^{18}$. On y raconte la rencontre de deux êtres qui ne se connaissent pas, Anna et l'homme, et qui semblent attendre pendant les quelques heures où ils seront réunis que quelque chose entre eux se produise. Le résumé même de l'histoire apparaît difficile à faire, tant la situation livre peu sa raison d'être et sa signification. Qui sont cet homme et cette femme? Qu'attendent-ils l'un et l'autre de cette rencontre? Qu'adviendra-t-il de ce moment vécu en commun ? Le récit décrit un morceau de vie sans lui attribuer de perspective d'ensemble et d'explication. Tel qu'il se donne à lire, Anna à la lettre $C$ se ramène donc à une succession de menus faits : l'homme est chez Anna, elle sert du thé glacé, ils vont s'asseoir sur le balcon, ils observent un groupe de gens occupés à leur cours de tai-chi, Anna et l'homme se parlent peu, hésitent à se regarder, l'homme désire Anna, celle-ci prépare le repas, ils mangent, la chaleur de l'été les oppresse, etc.

Curieusement, c'est bien une narration omnisciente qui prend en charge ce récit dominé par un tel sentiment d'incomplétude. Non représenté, hétérodiégétique, le

17 Dominique RABATÉ, de même que Jean-Yves TADIÉ, se sont intéressés au récit, sans cependant inclure ses manifestations contemporaines (Dominique Rabaté, Vers une littérature de l'épuisement, Paris, José Corti, 1991 ; Jean-Yves Tadié, Le récit poétique, Paris, Gallimard, coll. «Tel », 1994).

18 Voir à ce sujet: Frances FORTIER, Andrée MERCIER et al., "Trois récits, un stylème : les contours d'un genre ", dans Richard Saint-Gelais (dir.), Nouvelles tendances en théorie des genres, Québec, NB Université, 1998, p. 227-267. 
narrateur montre d'entrée de jeu son rôle très fin d'observateur. Il décrit minutieusement les faits et gestes, autant qu'il signale les sentiments éprouvés par les personnages :

Anna ouvre le frigo, prend un pot en verre bleu rempli de thé froid où surnage une tranche de citron. Ouvre l'armoire située au-dessus du frigo, se met sur la pointe des pieds, étire le bras et attrape, du bout des doigts, deux verres du même bleu translucide. Verse le thé brunâtre, en offre un à l'homme. Elle se trouve subitement ridicule. Déteste jusqu'à la nausée le thé qu'elle a décidé de boire jusqu'au fond du verre. (p. 8)

À première vue, on pourrait croire que le narrateur délègue la focalisation alternativement à ses deux personnages, sans véritablement s'approprier la perception, ce qui expliquerait en partie un savoir incomplet. Certains passages montrent bien toutefois l'absence de foyer focal défini, au profit d'une perspective générale redevable à l'instance abstraite de narration («Tous les deux, accroupis, observent les pains décongeler derrière la vitre de la cuisinière. La tendresse s'insinue entre eux. Sans qu'elle soit appelée. Logée » p. 36). Globalement, le regard du narrateur passe d'un personnage à l'autre, les recouvre tous deux parfois, montre la femme observant l'homme, et vice versa. Bref, la vision oscille sans jamais s'ordonner strictement au regard des personnages. On doit donc parler de focalisation zéro, ce qui correspond d'ailleurs davantage au fonctionnement habituel d'une narration omnisciente.

Description attentive, accès à l'intériorité des personnages, point focal indéterminé, d'où surgit dès lors le sentiment d'incomplétude très marqué de ce récit ? Il apparaît sans doute dès les premiers mots du texte qui plongent le lecteur dans une situation déjà en cours où sont engagés des personnages qui ne seront jamais introduits : non pas un chat, une jeune femme et un homme, mais le chat, Anna et l'homme. Le recours à la narration simultanée enlève également toute perspective temporelle au récit : le narrateur suit fidèlement le cours immédiat des événements sans anticiper ni revenir sur le passé. La présentation matérielle du texte exprime cependant avec le plus d'évidence les vides du récit. Celui-ci se donne 
comme une suite de tableaux ostensiblement détachés les uns des autres par les blancs de la page. Le livre prend l'allure d'un recueil et, à l'inverse du roman d'Echenoz qui ne comportait aucun chapitre ni saut de pages, multiplie les ruptures, crée des ellipses et des arrêts entre les scènes, alors même pourtant qu'il constitue une suite temporelle tout à fait continue. En effet, l'espace entre chaque tableau se ramène tout au plus à quelques minutes, parfois aucun saut temporel ne semble même perceptible, comme si l'espace typographique servait à découper en instants d'émotion une suite événementielle qui posséderait, sinon, peu de relief.

Si traditionnellement la narration omnisciente cherche à combler les vides de l'histoire en multipliant les foyers de focalisation, en complétant par les anachronies les limites de sa séquence temporelle, elle semble servir ici à montrer les interstices. Alors que le narrateur d'Echenoz montre l'incompétence cognitive de son personnage et y supplée, en révélant par exemple l'énigme du bruit des balles de golf ou en poursuivant la description des intérieurs d'automobiles quand Victoire s'en désintéresse, celui de Tremblay ne s'autorise pas à aller au-delà d'un certain seuil de compréhension. Le regard intime ou distancié qu'il jette sur ses personnages, décrit mais sans vraiment expliquer. L'émotion éprouvée sera dite, par exemple, sans que sa cause profonde soit donnée. Anna, à certains moments, éprouve du désir pour l'homme, plus tard, elle aura pour lui de la tendresse, elle le haïra aussi. D'un état à l'autre toutefois, les blancs du texte viennent montrer les vides, l'absence de logique, l'incompréhensible cours des choses et des sentiments. Il semble ici que l'omniscience ne s'autorise pas à aller plus loin que la vie ellemême, qu'elle refuse de donner à l'existence un sens qu'elle ne possède pas. La compétence cognitive du narrateur est régie par celle des personnages : ceux-ci vivent leur incompétence, ne comprennent pas les gestes qu'ils posent ni ce qu'ils éprouvent; le narrateur, quant à lui, se sert de son autorité non pour interpréter les événements, mais pour les montrer tels qu'ils sont vécus. 
On se rappellera que le roman d'Echenoz révèle avec fracas, à la dernière page, le «trou » inattendu de la narration. La relecture du roman montre une omniscience mystificatrice qui manifeste son autorité tout en protégeant ses arrières. Ainsi en est-il des passages où Victoire rencontrera LouisPhilippe (le présumé suicidé) : le narrateur semblera en déléguer la focalisation à Victoire, témoin à la fiabilité douteuse, à l'état psychologique plutôt énigmatique. Celle-ci n'a-t-elle pas été victime d'hallucinations, pourrait alors se demander le lecteur? Nulle réponse bien sûr, mais l'invitation à rétablir un semblant de cohérence en reportant la responsabilité de l'embrouillamini final sur les épaules de Victoire. Si le lecteur ne peut accuser le narrateur de mensonge délibéré, il doit admettre que l'information a été transmise malicieusement. Force détails sont livrés, à défaut en somme des points essentiels de l'affaire ; pensons à la description du pavillon que louera Victoire et qui donnera lieu à un inventaire minutieux des moindres objets :

Plus tard elle venait d'inspecter le pavillon en détail, d'ouvrir les penderies vides où s'entrechoquaient des cintres et les tiroirs pleins d'objets incomplets : albums photographiques désaffectés, clefs sans étiquette, cadenas sans clefs, manches d'accessoires et poignées de portes, tronçons de bougies, fragments de montants de lit, montre privée de sa grande aiguille. (p. 21)

Cet amas de petits détails cache évidemment les vides d'un récit qui se révélera semblable à ces multiples objets, tous incomplets. La gestion singulière du savoir est ici ludique et contourne la convention de l'omniscience pour tromper le lecteur et lui proposer de combler les interstices. Le roman subséquent d'Echenoz, Je m'en vais, montre d'ailleurs que la cohérence peut être rétablie.

Le récit de Tremblay contient aussi des vides, il les exhibe toutefois tout au long de sa progression. Son narrateur, plus explicitement que celui d'Echenoz, manifeste une omniscience tronquée qui peut conduire le lecteur à chercher lui-même le sens de la rencontre d'Anna et de l'homme. Il reste que le narrateur de Tremblay semble prendre acte de l'incohérence fondamentale de la vie, qu'il indexe à 
son récit les ruptures de signification qui ponctuent le cours des choses. L'entreprise n'a rien de ludique et met en doute la possibilité de trouver une réponse, comme s'il importait plutôt de montrer tout ce qui échappe à la compréhension. L'omniscience ne sert pas, comme dans Un an, à mystifier le lecteur, à le tromper en cachant une partie des faits, elle sert apparemment à démystifier l'image d'un monde compris et contrôlé.

$* * * *$

On le voit, la narration omnisciente opère, dans certains textes contemporains, une gestion très particulière du savoir. La convention d'une instance toute puissante est utilisée mais pour être bousculée, détournée, et pour confier au lecteur une large part du travail d'interprétation. En somme, le narrateur joue à fond son rôle d'observateur mais beaucoup moins celui d'interprète. Dans Un an, le détournement de la narration omnisciente est au service de l'œuvre, plus précisément d'un style fondé sur l'utilisation de modèles romanesques et sur le jeu. Un an commence comme un roman policier (mort suspecte et fuite) qui, plutôt que de résoudre l'énigme initiale qu'il pose, aboutira à une énigme encore plus insoluble. Le lecteur se sentira trompé par ce narrateur toutpuissant, mais tout-puissant à la façon d'Echenoz. Dans Anna à la lettre $C$, l'omniscience sera mise au service d'un genre, au prix aussi de son détournement. Le récit, en effet, ne saurait admettre l'omniscience sans la soumettre à ses propres enjeux plus près du témoignage intime, du regard subjectif porté sur le monde, que du discours souverain. Il ne s'agira donc pas de tromper le lecteur en le privant d'une interprétation possible, mais de lui montrer que l'interprétation n'existe pas.

Peut-on parler ici d'une esthétique contemporaine ? Si les deux exemples ne suffisent pas à rendre compte des diverses manifestations des formes narratives, ils témoignent tout à fait d'un retour du récit, prenant acte de l'examen critique des décennies précédentes. Moins spectaculaire que les jeux polyphoniques qui, dans de nombreuses œuvres, 
déstabilisent la voix narrative et son identité, la narration omnisciente, tant dans Un an que dans Anna à la lettre $C$, participe elle aussi à ce que Dominique Viart a nommé « la pérennité du soupçon ${ }^{19}$. Il est vrai que, autorité toutepuissante s'il en est, une telle convention pouvait difficilement sortir intacte de cette remise en cause des savoirs. Après s'être imposée, la narration omnisciente est donc à nouveau objet de suspicion. Les textes étudiés montrent bien, cependant, comment les formes narratives actuelles sont appelées à concilier une action «à la fois démystificatrice et mythifiante », pour reprendre les propos de Frédéric Tristan ${ }^{20}$. En effet, si Un an et Anna à la lettre C, sous des modalités différentes, manifestent l'action démystificatrice d'une fiction qui «ne s'embarrassant en rien des apparences du réel est capable de déstabiliser les conventions, les conformismes », ils rappellent que cette action est également «[m]ythifiante parce qu'à son plus haut niveau, le récit est cette seule expression du réel susceptible de ne pas mentir et d'atteindre le centre vivant de l'imaginaire : le merveilleux $»^{21}$.

19 Dominique VIART, op. cit., p. 11.

20 Frédéric TRISTAN désigne ainsi plus largement le retour du récit après sa mise en cause plus radicale par la modernité. "Le spectacle est à l'intérieur ", entretien avec Jean-Luc Moreau, dans Jean-Luc Moreau, La nouvelle fiction, Paris, Critérion, 1992, p. 87-88, cité par Dominique Viart, op. cit., p. 17.

21 Ibid. 